\title{
ESTUDIO BIOESTRATIGRÁFICO Y PALEOAMBIENTAL DE LA FORMACIÓN RÍO TURBIO (EOCENO MEDIO-SUPERIOR) EN EL SUDOESTE DE PATAGONIA (ARGENTINA) BASADO EN QUISTES DE DINOFLAGELADOS
}

\author{
M. SOL GONZÁLEZ ESTEBENET \& G. RAQUEL GUERSTEIN \\ Instituto Geológico del Sur, Universidad Nacional del Sur, Departamento de Geología, CONICET, San Juan 670, \\ B8000ICN Bahía Blanca, Argentina.sol.gonzalezestebenet@uns.edu.ar, raquel.guerstein@uns.edu.ar \\ SILVIO CASADÍO \\ Universidad Nacional de Río Negro, CONICET, Av. Roca 1242, 8332 General Roca, Río Negro, Argentina. \\ scasadio@conicet.gov.ar
}

\begin{abstract}
BIOSTRATIGRAPHIC AND PALEOENVIRONMENTAL STUDY OF RÍO TURBIO FORMATION (MIDDLE-UPPER EOCENE) IN SOUTHWEST OF PATAGONIA (ARGENTINA) BASED ON DINOFLAGELLATE CYSTS. During the middle and late Eocene the Austral Basin, in southern Patagonia (Argentina), was flooded by an Atlantic Ocean transgression, which was responsible for the deposition of the upper member of the Río Turbio Formation. We have analyzed the dinoflagellate cyst assemblages from this stratigraphic section in order to provide a biostratigraphic framework and reconstruct the paleogeographic and paleoceanographic conditions in this area. Dinoflagellate cyst events recorded were compared and interpreted based taking into account other records recognized for the South Pacific Ocean. The comparison allowed us to suggest an age between 45.5 Ma (mid-Lutetian) and 33.5 Ma (Priabonian) for the upper member of the Rio Turbio Formation. We recognized three zones of dinoflagellate cysts. Zone I presents an alternating dominance between Enneadocysta dictyostila, Deflandrea antarctica and Vozzhennikovia apertura indicating two sea level rises. Zone II is dominated by $V$. apertura suggesting high trophic levels and cool waters in a shallow-marine coastal environment. The lower part of the Zone III shows a high abundance of Turbiosphaera filosa with different morphotypes, which may correspond to physicochemical changes in the water column. The uppermost part of the Zone III is characterized by Protoperidinaceae and typical forms of T. filosa suggesting an oceanic environment influenced by upwelling processes. Zones I and II are defined by the dominance of middle Eocene endemic-Antarctic assemblage, while Zone III shows a significant replacement of these taxa by cosmopolitan species. This turnover seems to be forced by the deepening of the Southern Atlantic Ocean gateways and the changes in the ocean circulation patterns.
\end{abstract}

Key words: dinoflagellate cysts, middle-late Eocene, Drake Passage, biostratigraphy, paleoenvironment.

RESUMO - Durante o Eoceno médio e final, a bacia Austral, no sul da Patagônia (Argentina), esteve sujeita a uma transgressão do Oceano Atlântico, responsável pela deposição do membro superior da Formação Río Turbio. Assembleias de cistos de dinoflagelados desta porção da Formação Río Turbio foram analisadas a fim de promover a construção de um esquema bioestratigráfico e a reconstrução das condições paleogeográficas e paleoceanográficas nesta área. Eventos registrados relacionados a cistos de dinoflagelados da Formação Río Turbio foram comparados e interpretados considerando outros análogos reconhecidos para o sul do Oceano Pacífico. As comparações permitem sugerir uma idade entre 45,5 Ma (Lutetiano médio) e 33,5 Ma (Priaboniano) para o membro superior da Formação Río Turbio. Três zonas de cistos de dinoflagelados foram estabelecidas. A Zona I apresenta alternância na dominância de Enneadocysta dictyostila, Deflandrea antarctica e Vozzhennikovia apertura, indicando duas elevações relativas do nível do mar. A Zona II é dominada por $V$. apertura, sugerindo níveis tróficos elevados e águas frias em ambiente marinho costeiro raso. A porção inferior da Zona III mostra abundância de Turbiosphaera filosa, com diferentes morfotipos, os quais podem corresponder a mudanças fisioquímicas na coluna d'água. A porção superior da Zona III é caracterizada por Protoperidinaceae e formas típicas de T. filosa, sugerindo ambiente oceânico influenciado por processos de ressurgência. As zonas I e II são definidas pela dominância de assembleias endêmicas do Mioceno médio da Antártica, enquanto a Zona III apresenta significativa substituição destes táxons por espécies cosmopolitas. Esta mudança parece ter sido forçada pelo aprofundamento das passagens do Oceano Atlântico Sul e por alterações nos padrões de circulação oceânica.

Palavras-chave: cistos de dinoflagelados, Eoceno Médio-Superior, Passagem de Drake, bioestratigrafia, paleoambiente. 


\section{INTRODUCCIÓN}

Los dinoflagelados son organismos unicelulares, abundantes y diversos en ambientes marinos proximales y neríticos. En su fase plantónica son susceptibles a las fluctuaciones de temperatura, salinidad, disponibilidad de nutrientes del agua superficial y profundidad del agua (Dale, 1996). Muchos de ellos producen quistes de dinoflagelados de pared orgánica que se preservan en el registro fósil con una amplia distribución en sedimentos de ambientes de plataforma. Los quistes de dinoflagelados representan una herramienta valiosa en la determinación de las condiciones físicas y químicas de las aguas superficiales en el pasado y complementan la información brindada por otros grupos de microfósiles. En particular, han demostrado ser sumamente útiles en la reconstrucción de paleoambientes marinos del Paleógeno (Sluijs et al., 2005 y referencias allí citadas) y en la resolución de problemas estratigráficos (Bijl et al., 2013a).

Durante el Paleógeno ( 65-35 Ma) en el Hemisferio Sur se reconocen importantes cambios paleogeográficos, entre ellos el desarrollo del Pasaje de Drake y el Conducto de Tasmania, los cuales generaron modificaciones importantes en la circulación oceánica como el establecimiento de la Corriente Circumpolar Antártica (Scher \& Martin, 2006; Stickley et al., 2004a; Lagabrielle et al., 2009; González Estebenet et al., 2014a) y la formación de aguas intermedias relativamente frías (Bijl et al., 2013b). En este período se produjo la transición de un clima cálido hacia uno frío con intervalos cálidos de corta duración o hipertermales, como por ejemplo el Óptimo Climático del Eoceno Medio (Zachos et al., 2008; Bohaty \& Zachos, 2003; Bohaty et al., 2009; Bijl et al., 2010).

Como respuesta a las variaciones en la temperatura del agua superficial y a los patrones de circulación oceánica que ocurrieron durante este intervalo, las asociaciones de quistes de dinoflagelados experimentaron modificaciones sustanciales. En el Paleoceno y Eoceno temprano las asociaciones de dinoflagelados se encuentran dominadas por taxa cosmopolitas típicos del Paleógeno temprano, mientras que en el Eoceno medio ( $\sim 50 \mathrm{Ma})$ las asociaciones muestran un cambio significativo con predominio de especies endémicas de altas latitudes del Hemisferio Sur (Bijl et al., 2011; 2013a,b) conformando una asociación originalmente denominada "Flora Transantárctica" por Wrenn \& Beckmann (1982). Esta asociación endémica-antártica continuó su desarrollo hasta la transición Eoceno-Oligoceno, cuando se produjo la extinción de la mayoría de los miembros de dicha asociación, los que fueron reemplazadas por taxa cosmopolitas (Sluijs et al., 2003; Stickey et al., 2004; Bijl et al., 2011; Houben et al., 2013). La extinción de la asociación endémica podría haber sido una consecuencia de la profundización del Pasaje de Drake y el Conducto de Tasmania, y de las subsecuentes modificaciones en los patrones de circulación oceánica (Sluijs et al., 2003; Stickley et al., 2004a; Guerstein et al., 2008a; Houben et al., 2011, 2013). Los cambios en las asociaciones de quistes de dinoflagelados paleógenas han sido calibrados en la plataforma de Tasmania con la Escala de Polaridad Geomagnética de Vandenberghe et al. (2012) usando magnetoestratigrafía y estratigrafía basada en isótopos estables (Bijl et al., 2013a). Según estos autores, el conjunto de datos de referencia de quistes de dinoflagelados puede ser aplicado a todo el Océano Austral brindando una importante herramienta para estudios bioestratigráficos.

La Patagonia extrandina se vio afectada por sucesivas trangresiones atlánticas durante el Cenozoico (Malumián \& Nañez, 2002). En particular, en el Eoceno medio a tardío tuvo lugar un ciclo transgresivo-regresivo que inundó gran parte de la cuenca Austral, durante el cual se depositó en el sudoeste de la provincia de Santa Cruz, el miembro superior de la Formación Río Turbio (FRT). Debido a la proximidad de la cuenca Austral al Pasaje de Drake, los depósitos marinos de la FRT constituyen una valiosa fuente de información para evaluar los cambios paleoambientales y paleoclimáticos ocurridos durante el Eoceno en el extremo sur de Sudamérica.

En este trabajo se analizan las asociaciones de quistes de dinoflagelados del miembro superior de la FRT que aflora en cercanías de la estancia Cancha Carrera, próxima a la localidad de Río Turbio (Figura 1). El objetivo es establecer un marco bioestratigráfico tomando como referencia los rangos de quistes de dinoflagelados calibrados con magnetoestratigrafía en testigos de la plataforma de Tasmania (Brinkhuis et al., 2003a; Sluijs et al., 2003; Stickey et al., 2004a; Bijl et al., 2013a) y reconstruir las condiciones paleoambientales en el sudoeste de la cuenca Austral durante el Eoceno medio a tardío.

\section{MARCO GEOLÓGICO}

En el margen más austral de la placa sudamericana (entre los $47^{\circ} \mathrm{S}-54^{\circ} \mathrm{S}$ ) se desarrolló durante el Cretácico TardíoNeógeno un cinturón plegado hacia el oeste y una cuenca de antepaís hacia el este, conocida como cuenca Austral o Magallanes, donde se depositaron unidades marinas y continentales (Biddle et al., 1986). En el sector occidental de la cuenca, al sudoeste de la provincia de Santa Cruz, se reconocen sedimentitas eocenas asignadas a la FRT. Esta formación se divide en dos miembros informales, el inferior y el superior. El miembro inferior, asociado al límite Eoceno temprano a medio (Guerstein et al., 2010), se dispone en discordancia sobre la Formación Cerro Dorotea, depositada en un ambiente marino somero durante el Paleoceno (Malumián et al., 2000; Malumián, 2002). El miembro superior de la FRT, se considera de edad Eoceno medio tardío-Eoceno tardío temprano (Kraemer et al., 2002; Malumián, 2002; Ramos, 2005; Casadío et al., 2009; González Estebenet et al., 2014b) y por encima de una discordancia erosiva se reconocen depósitos continentales de edad Eoceno tardío-Oligoceno temprano de la Formación Río Guillermo (Arguijo \& Romero, 1981; Ramos, 2005).

El miembro superior de la FRT representa la sedimentación en ambientes submareales correspondientes a un período transgresivo iniciado en el Eoceno medio tardío (Malumián, 2002) el cual está caracterizado por un nivel glauconítico muy extendido dentro de la Cuenca Austral (Calegari et al., 1993). Dicho nivel glauconítico permite correlacionar al miembro superior de la FRT con la Formación Man Aike en 


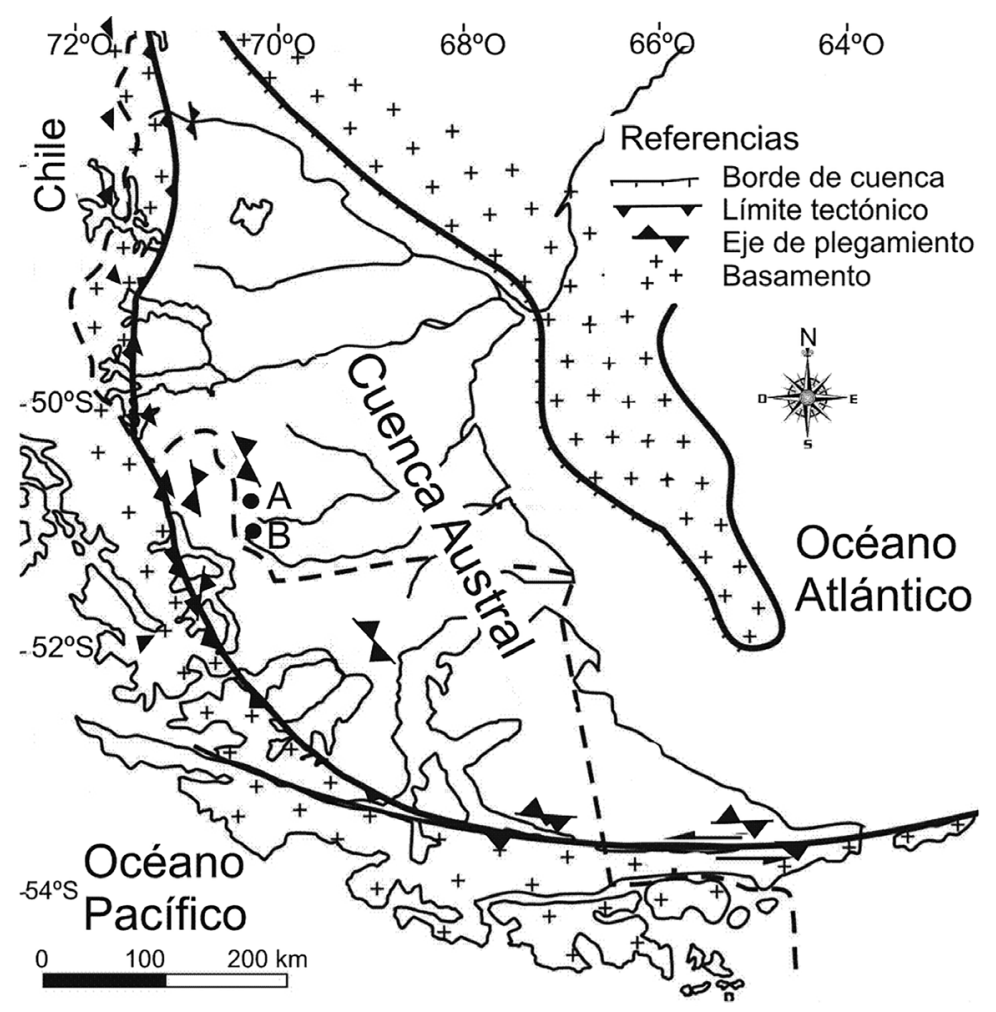

Figura 1. Mapa del sur de Patagonia, Argentina, modificado de Nullo et al. (1999). Extensión de la Cuenca Austral mostrando afloramientos del miembro superior de la Formación Río Turbio en Ea. Cancha Carrera (A, este trabajo) y sobre la Ruta Nacional 40 (B).

Figure 1. Map of the south of Patagonia, Argentina, modified from Nullo et al. (1999). Austral Basin extension showing outcrops of the Río Turbio Formation in Ea. Cancha Carrera (A, this paper) and Ruta Nacional 40 (B).

la Provincia de Santa Cruz (Concheyro, 1991; Malumián, 1990; Casadío et al., 2009; Guerstein et al., 2014), con la Formación Leticia (Olivero \& Malumián, 1999; Guerstein et al., 2008b), la sección inferior de la Formación Cerro Colorado y el Glauconítico B en la Provincia de Tierra del Fuego y con la Formación Ballena en Chile (Malumián 2002; Olivero \& Malumián, 2008). Los primeros estudios sobre quistes de dinoflagelados de la FRT fueron realizados por Archangelsky $(1968,1969)$ en testigos perforados por Yacimientos Carboníferos Fiscales (YCF). Recientemente Guerstein et al. (2014) y González Estebenet et al. (2014b) estudiaron las asociaciones de quistes de dinoflagelados del miembro superior de la FRT a partir de perfiles aflorantes sobre la Ruta Nacional 40.

\section{MATERIAL Y MÉTODOS}

Los materiales analizados provienen de cuatro secciones integradas en una columna de $410 \mathrm{~m}$ de espesor que afloran al sudoeste de la provincia de Santa Cruz, en las inmediaciones de la estancia Cancha Carrera al norte de la localidad minera de Río Turbio (51 $14^{\prime} 34^{\prime \prime} \mathrm{S}, 72^{\circ} 15^{\prime} 26^{\prime \prime} \mathrm{O}$; Figura 2). El marco estratigráfico para este trabajo se basa en Rodríguez Raising (2010), quién estudió las secciones aflorantes de la FRT y propuso un esquema estratigráfico secuencial reconociendo nueve secuencias depositacionales (SI-SIX). Las secuencias I y II pertenecen al miembro inferior de la FRT, mientras que las secuencias III-IX corresponden al miembro superior de dicha formación, de donde provienen las muestras palinológicas analizadas en este trabajo. Se obtuvo un total de 17 muestras y su ubicación estratigráfica se ilustra en la Figura 2. En todos los casos, el tratamiento de extracción fisicoquímica consistió en la eliminación de la fracción inorgánica con ácidos clorhídrico y fluorhídrico. La fracción orgánica se concentró mediante filtrados con tamices de 10, 25 y 180 $\mu \mathrm{m}$. Los residuos fueron teñidos con Bismarck C y montados en gelatina-glicerina. El procesamiento palinológico fue realizado en el Museo Argentino de Ciencias Naturales, Bernardino Rivadavia, Buenos Aires y los preparados fueron archivados en el Laboratorio de Palinología, Instituto Geológico del Sur, Bahía Blanca (LPUNS).

Para cada muestra se contó un mínimo de 200 quistes de dinoflagelados identificados a nivel de especie, a excepción de las muestras de CC 2 y CC 13, para los cuales se contaron sólo 150 ejemplares debido a su baja abundancia. El estudio taxonómico se realizó utilizando un microscopio óptico Nikon Eclipse $600\left(n^{\circ}\right.$ 772751) en el INGEOSUR, Departamento de Geología de la Universidad Nacional del Sur. Los taxones fueron asignados de acuerdo a Fensome et al. (2008) y Sluijs et al. (2009). Las fotos fueron tomadas con una cámara digital Micrometrics. Los ejemplares ilustrados se identificaron con el número de muestra y las referencias England Finder (EF). 

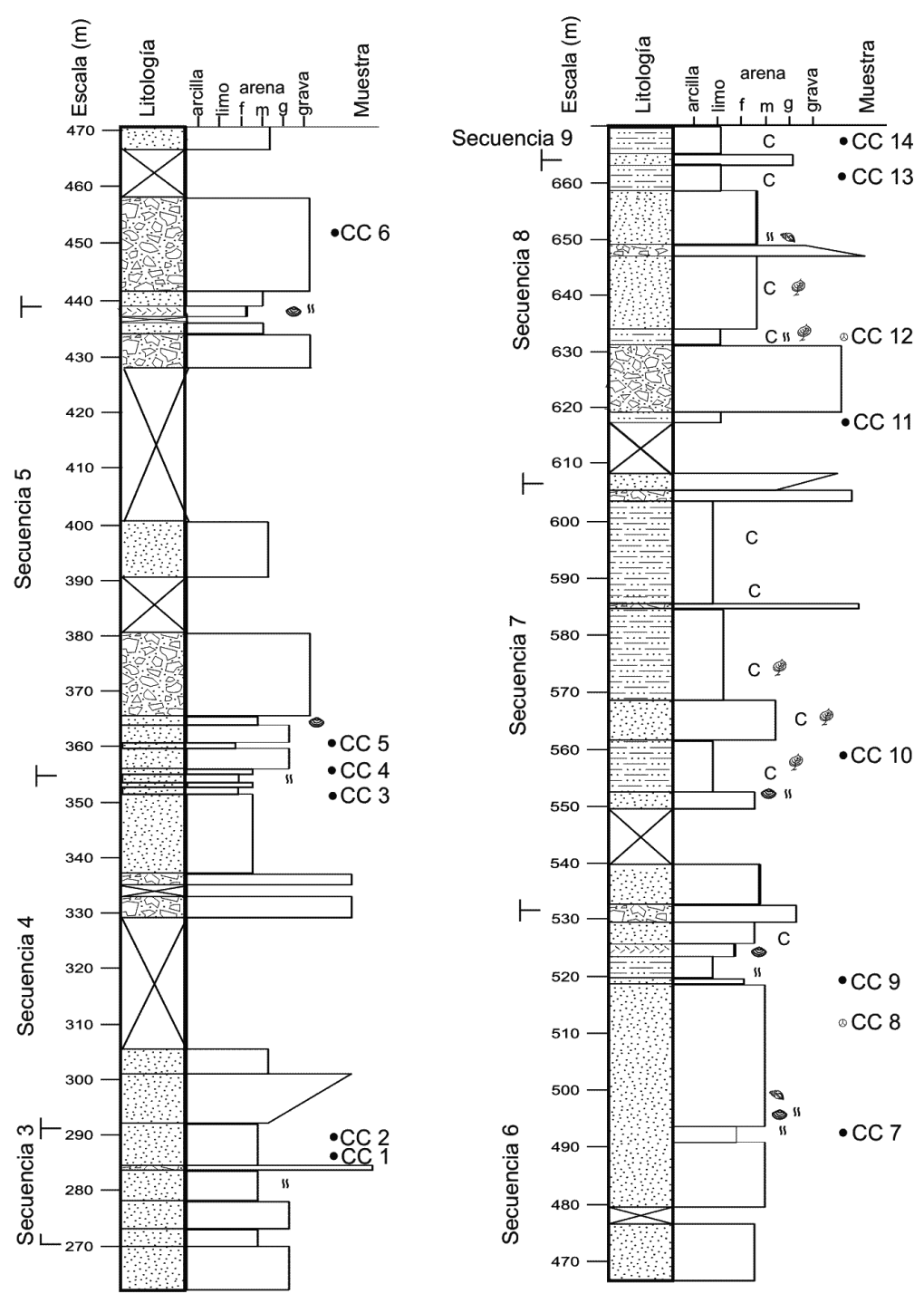

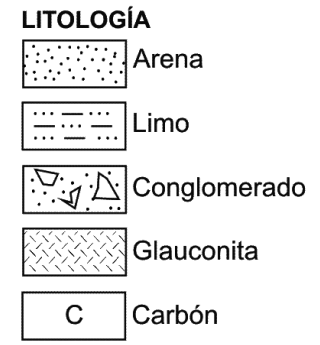

ESTRUCTURAS

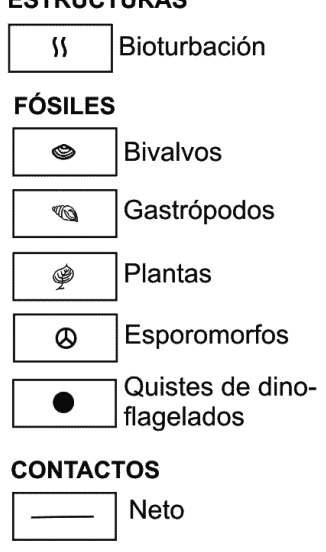

Figura 2. Perfil estratigráfico del miembro superior de la Formación Río Turbio aflorante en la Ea. Cancha Carrera con la ubicación de las muestras analizadas.

Figure 2. Stratigraphical section of the upper member of Río Turbio Formation cropping out in Ea. Cancha Carrera indicating sample numbers. Lithology: sandstone, siltstone, conglomerate, glauconite, coal. Sedimentary structures: bioturbation. Fossils: bivalves, gastropods, plants, sporomorphs, dinoflagellate cysts. Contacts: sharp.

El porcentaje de quistes peridinoideos (quistes-P) sobre el total de quistes de dinoflagelados se utilizó para estimar la productividad (Brinkhuis et al., 1998; van Mourik \& Brinkhuis, 2000; Crouch et al., 2003, Bijl, et al., 2010). También se calculó el porcentaje de quistes de dinoflagelados endémicos sobre el total de quistes de dinoflagelados con el objeto de caracterizar la temperatura del agua de mar superficial y el porcentaje de quistes de dinoflagelados/total de palinomorfos, a fin de estimar la proximidad a la línea de costa. Se realizó un análisis de componentes principales (biplot) a partir de una matriz de correlación utilizando el programa PAST, versión 2.17 (Hammer et al., 2001). La escala de tiempo geológico utilizada es la propuesta por Vandenberghe et al. (2012).

\section{RESULTADOS}

Del total de 17 muestras analizadas en el miembro superior de la FRT, 12 muestras contienen altas concentraciones de quistes de dinoflagelados marinos (CC 1 a CC 7, CC 9 a CC 11, CC 13 y CC 14), 2 muestras poseen sólo palinomorfos no marinos, principalmente polen, esporas y zigósporas de algas verdes, características de agua dulce (CC 8 y CC 12) y 3 muestras ubicadas en la porción superior del perfil resultaron estériles (CC 15, CC 16 y CC 17). En la Tabla 1 se muestra un total de 24 especies registradas, el orden al cual pertenecen y la distribución biogeográfica (endémica, bipolar o cosmopolita) de acuerdo a Bijl et al. (2013b) y Houben et al. (2013). La mayoría de los ejemplares se ilustran en la Figura 3. La composición cuantitativa de las asociaciones de 
Tabla 1. Lista de especies de quistes de dinoflagelados citadas en el texto. La distribución latitudinal se basa en Bijl et al. (2011) y Houben et al. (2013).

Table 1. List of species of dinocysts cited in the text. The latitudinal distribution is based on Bijl et al. (2011) and Houben et al. (2013).

\begin{tabular}{|c|c|c|}
\hline Especies de quistes de dinoflagelados & Orden & Distribución latitudinal \\
\hline Achomosphaera sp. A & Gonyaulacales & cosmopolita? \\
\hline Deflandrea antarctica Wilson, 1967a & Peridiniales & endémica-antárctica \\
\hline Deflandrea cf. D. granulata Menéndez, 1965 & Peridiniales & endémica-antárctica \\
\hline Enneadocysta brevistila Fensome et al., 2006 & Gonyaulacales & endémica-antárctica \\
\hline Enneadocysta dictyostila (Menéndez) Fensome et al., 2006 & Gonyaulacales & endémica-antárctica \\
\hline Hystrichosphaeridium truswelliae Wrenn \& Hart, 1988 & Gonyaulacales & cosmopolita \\
\hline Hystrichosphaeridium tubiferum (Ehrenberg) Deflandre, 1937b & Gonyaulacales & cosmopolita \\
\hline Impagidinium dispertitum (Cookson \& Eisenack) Stover \& Evitt, 1978 & Gonyaulacales & cosmopolita \\
\hline Impletosphaeridium sp. & Gonyaulacales & cosmopolita \\
\hline Lejeunecysta spp. & Peridiniales & cosmopolita \\
\hline Lingulodinium $\mathrm{sp}$. & Gonyaulacales & cosmopolita \\
\hline Nematosphaeropsis sp. & Gonyaulacales & cosmopolita \\
\hline Operculodinium centrocarpum (Deflandre \& Cookson) Wall, 1967 & Gonyaulacales & cosmopolita \\
\hline Operculodinium israelianum (Rossignol) Wall, 1967 & Gonyaulacales & cosmopolita \\
\hline Selenopemphix crenata Matsuoka \& Bujak, 1988 & Peridiniales & cosmopolita \\
\hline Selenopemphix nephroides Benedek, 1972 & Peridiniales & cosmopolita \\
\hline Spinidinium macmurdoense (Wilson) Lentin \& Williams, 1976 & Peridiniales & endémica-antárctica \\
\hline Spiniferites membranaceus (Rossignol) Sarjeant, 1970 & Gonyaulacales & cosmopolita \\
\hline Spiniferites mirabilis (Rossignol) Sarjeant, 1970 & Gonyaulacales & cosmopolita \\
\hline Spiniferites scalenus Guerstein et al., 2008b & Gonyaulacales & endémica-antárctica? \\
\hline Spiniferites ramosus (Ehrenberg) Mantell, 1854 & Gonyaulacales & cosmopolita \\
\hline Thalassiphora pelagica (Eisenack) Eisenack \& Gocht, 1960 & Gonyaulacales & cosmopolita \\
\hline Turbiosphaera filosa (Wilson) Archangelsky, 1968 & Gonyaulacales & cosmopolita \\
\hline Vozzhennikovia apertura (Wilson) Lentin \& Williams, 1976 & Peridiniales & endémica-antárctica \\
\hline
\end{tabular}

dinoflagelados en los distintos niveles estudiados y el biplot correlacionando muestras y especies con frecuencias mayores al $20 \%$ en al menos una muestra se ilustran en la Figuras 4 y 5 , respectivamente.

A partir de los esquemas de abundancias relativas (Figura 4) y del análisis de componentes principales (Figura 5) fue posible diferenciar tres biozonas:

Zona I: comprende las muestras CC 1 a CC 6, entre los 286 a $450 \mathrm{~m}$ medidos desde la base del perfil. Esta zona muestra una alternancia en el predominio de quistes de dinoflagelados típicos de la asociación endémica. Las muestras CC 1, CC 2 y CC 5 están dominadas por especies de Deflandrea, principalmente D. antarctica (33\%, 38\% y $43 \%$, respectivamente) complementadas por Enneadocysta dictyostila $(24 \%, 25 \%$ y $9 \%)$ y Vozzhennikovia apertura $(10 \%, 13 \%$ y $19 \%)$. Es importante mencionar que en las muestras CC 1 y CC 2 se registra la presencia de Hystrichosphaeridium truswelliae, un importante indicador bioestratigráfico. Thalassiphora pelagica se encuentra presente solamente en la muestra CC 1 alcanzando el $21 \%$ del total de quistes de dinoflagelados. Las muestras CC 3 y CC 6 muestran las máximas frecuencias de E. dictyostila ( $89 \%$ y $69 \%$ ), con una baja representación de $V$. apertura ( $1 \%$ en ambos casos). La muestra CC 4 se encuentra dominada por $V$. apertura $(59 \%)$ con un menor porcentaje de E. dictyostila (14\%).
Zona II: abarca el intervalo entre los 492 y $560 \mathrm{~m}$ desde la base del perfil y se encuentra representada por las muestras CC 7, CC 9 y CC 10, las que registran las máximas abundancias de Vozzhennikovia apertura $(87 \%, 97 \%, 85 \%$ respectivamente).

Zona III: comprende a las muestras CC 11, CC 12 y CC 14 entre $\sim 617$ y $670 \mathrm{~m}$ desde la base de la sección. Se caracteriza por altos porcentajes de especies cosmopolitas, entre ellas, Turbiosphaera filosa $(85 \%, 31 \%$ y $46 \%)$ y protoperidináceas pertenecientes a los géneros Selenopemphix y Lejeunecysta $(3,38$ y $13 \%)$.

\section{DISCUSIÓN}

\section{Bioestratigrafía}

La falta de herramientas precisas para datar y correlacionar las unidades sedimentarias de las cuencas del Océano Atlántico Sudoccidental constituye una limitante para interpretar la evolución paleoambiental y paleoceanográfica durante el Paleógeno. En este trabajo se compara la distribución de quistes de dinoflagelados en las secciones analizadas con los rangos bioestratigráficos de las especies de dinoflagelados diagnósticos (Brinkhuis et al., 2003b), las zonas de dinoflagelados de alta resolución del Pacífico Sur (South Pacific Dinocyst Zones, SPDZ) propuestas por Bijl et al. (2013a) para el Paleógeno y las asociaciones de quistes de dinoflagelados (Dinocyst association, DA) para el límite 

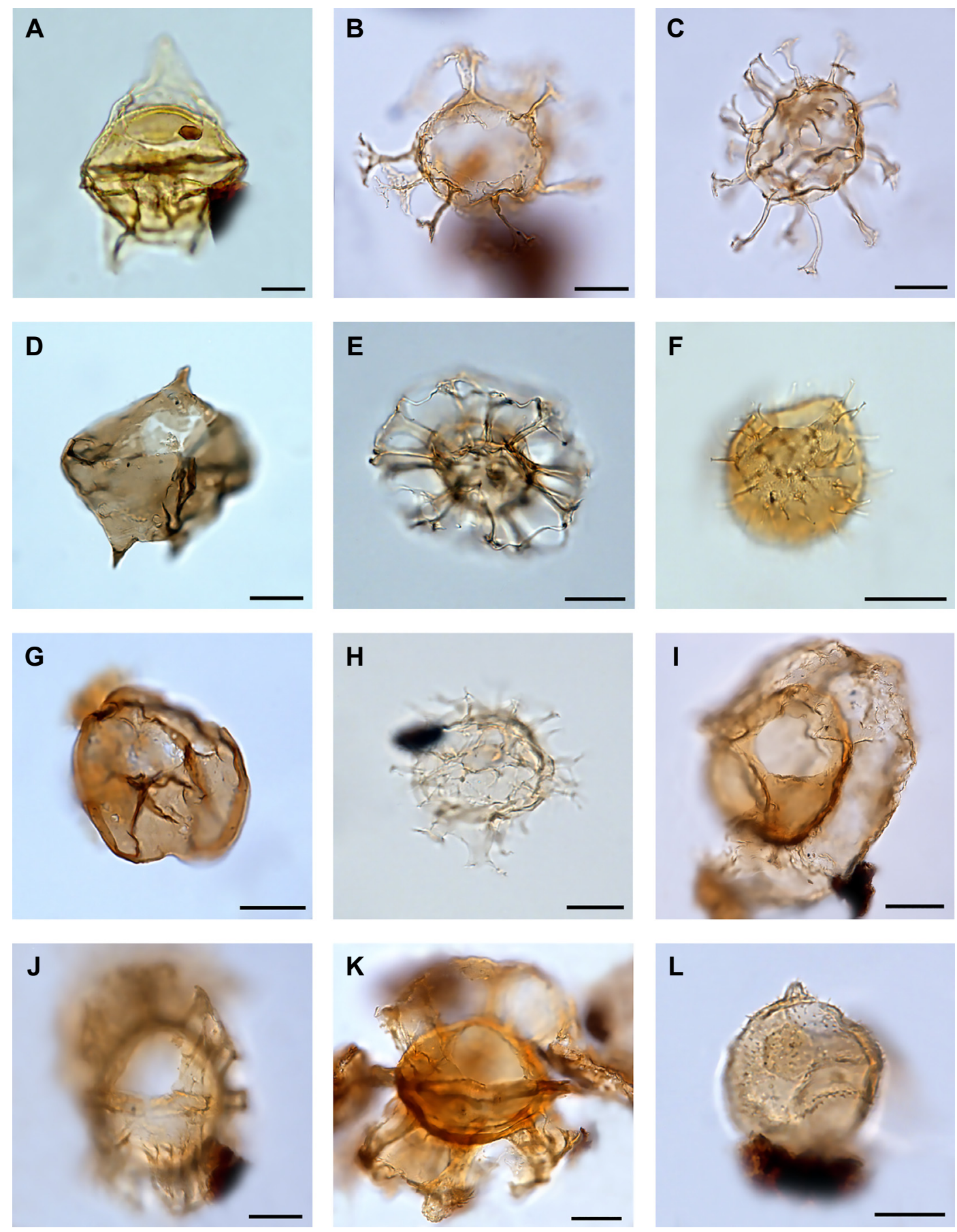

Figura 3. Quistes de dinoflagelados del miembro superior de la Formación Río Turbio en secciones aflorantes en Ea. Cancha Carrera. Los ejemplares se identifican mediante el número de muestra y referencias England Finder. A, Deflandrea antarctica Wilson, 1967a (CC 2/V35); B, Enneadocysta dictyostila (Menéndez) Fensome et al., 2006 (CC 6/Q45-2); C, Hystrichosphaeridium tubiferum (Ehrenberg) Deflandre, 1937b (CC 2/K44-2); D, Lejeunecysta sp. (CC 13/L 18-1); E, Nematosphaeropsis sp. (CC 13/U22-2); F, Operculodinium israelianum (Rossignol) Wall, 1967 (CC 14/H32); G, Selenopemphix nephroides Benedek, 1972 (CC 11/Y26-2); H, Spiniferites membranaceus (Rossignol) Sarjeant, 1970 (CC 13/S38); I, Thalassiphora pelagica (Eisenack) Eisenack \& Gocht, 1960 (CC 1/W40); J-K, Turbiosphaera filosa (Wilson) Archangelsky, 1969a; $\mathbf{J}$, procesos con un desarrollo temprano a intermedio (CC 11/T44-3); K, procesos con un desarrollo completo (CC 11/U30); L, Vozzhennikovia apertura (Wilson) Lentin \& Williams, 1976 (CC 10/V43-4). Escalas $=20 \mu \mathrm{m}$.

Figure 3. Dinoflagellate cysts of the upper member of Río Turbio Formation cropping out in Ea. Cancha Carrera. Specimens are identified by sample number and England Finder references. A, Deflandrea antarctica Wilson, 1967a (CC 2/V35); B, Enneadocysta dictyostila (Menéndez) Fensome et al., 2006 (CC 6/Q45-2); C, Hystrichosphaeridium tubiferum (Ehrenberg) Deflandre, 1937b (CC 2/K44-2); D, Lejeunecysta sp. (CC 13/L 18-1); E, Nematosphaeropsis sp. (CC 13/U22-2); F, Operculodinium israelianum (Rossignol) Wall, 1967 (CC 14/H32); G, Selenopemphix nephroides Benedek, 1972 (CC 11/Y26-2); H, Spiniferites membranaceus (Rossignol) Sarjeant, 1970 (CC 13/S38); I, Thalassiphora pelagica (Eisenack) Eisenack \& Gocht, 1960 (CC 1/W40); J-K, Turbiosphaera filosa (Wilson) Archangelsky, 1969a; J, early to intermediate stage in processes formation (CC 11/T44-3); K, complete stage in processes formation (CC 11/U30); L, Vozzhennikovia apertura (Wilson) Lentin \& Williams, 1976 (CC 10/V43-4). Scale bars $=20 \mu \mathrm{m}$. 


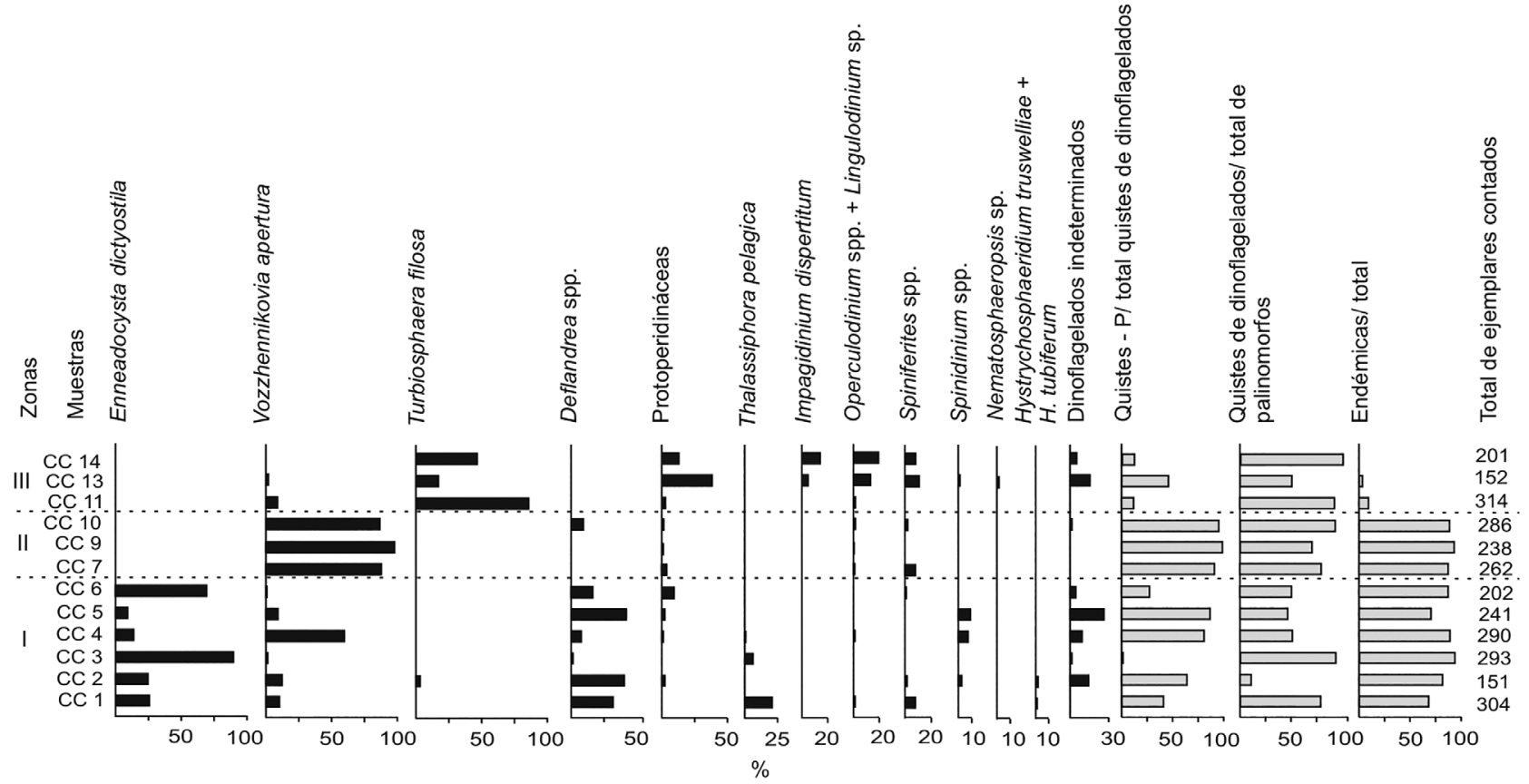

Figura 4. Distribución cuantitativa de quistes de dinoflagelados y zonas determinadas para el miembro superior de la Formación Río Turbio.

Figure 4. Zones and quantitative distribution of dinocysts from the upper member of the Río Turbio Formation.

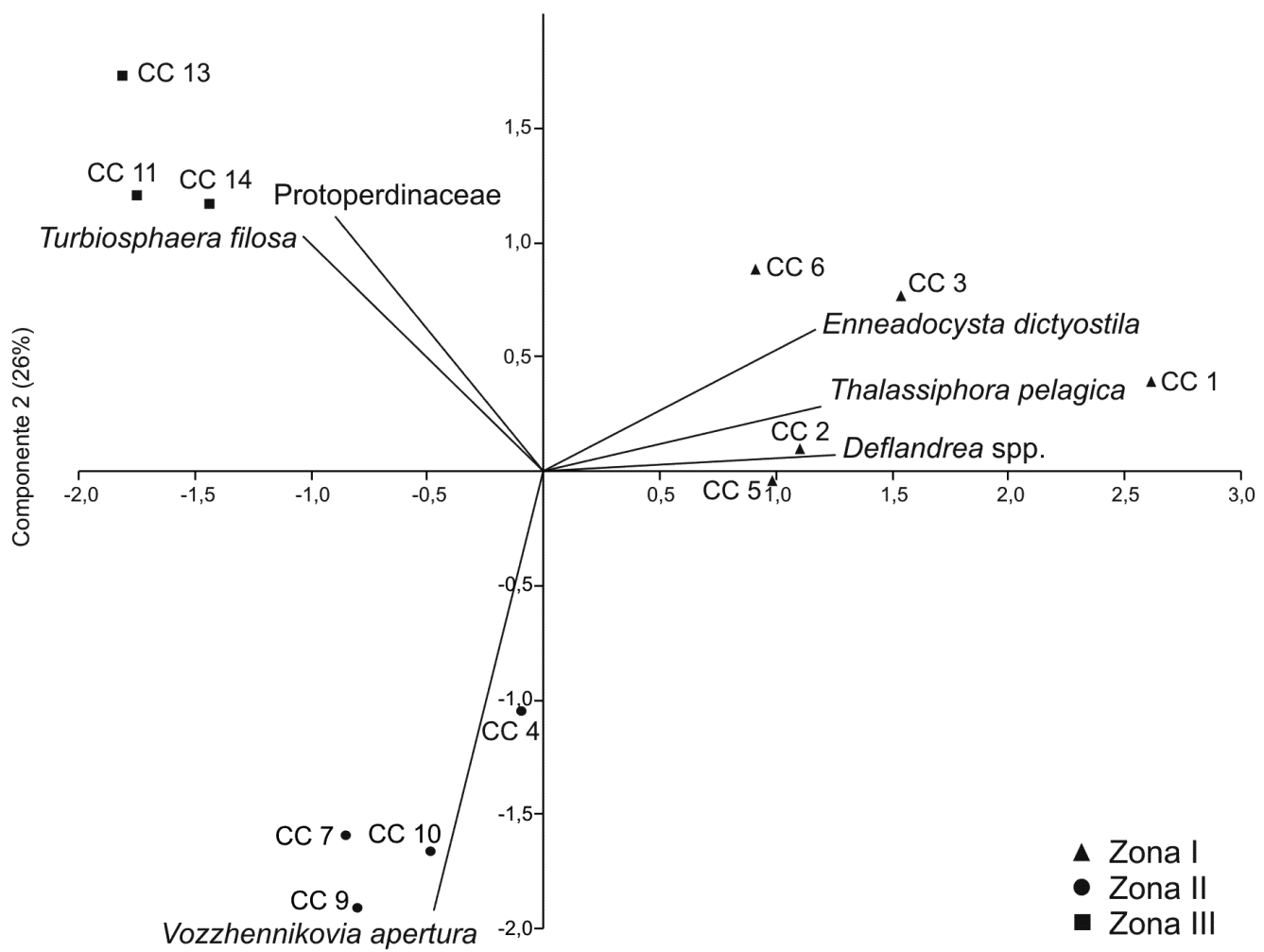

Componente $1(34 \%)$

Figura 5. Análisis de componentes principales mostrando la correlación entre las muestras y las especies con frecuencias mayores al $20 \%$ en al menos una muestra.

Figure 5. Principal component analysis showing the correlation between samples and species with frequencies higher than $20 \%$ in at least one sample. 
Eoceno tardío-Oligoceno determinadas por Sluijs et al. (2003) y Stickey et al. (2004a). Los registros utilizados para definir dichas zonaciones están calibrados con magnetoestratigrafía y estratigrafía de isótopos estables. Los rangos bioestratigráficos de las especies de dinoflagelados diagnósticos se basan en eventos de dinoflagelados tales como la primera ocurrencia: PO; la primera ocurrencia común: POC ( $>25 \%)$; la última ocurrencia: UO y la última ocurrencia común: UOC $(>25 \%)$. Aunque la mayoría de las especies de dinoflagelados hallados en el miembro superior de la FRT poseen rangos estratigráficos extensos, los eventos estratigráficos de algunas especies permiten establecer una edad máxima y mínima para estos depósitos (Figura 6).

En la asociación de quistes de dinoflagelados de CC 1, la base de la Zona I se registra la PO de Enneadocysta dictyostila, ya que en las muestras analizadas de las secuencias inferiores correspondientes al miembro inferior de la FRT, no se observa la presencia de dicha especie (Guerstein, obs. pers.). En las investigaciones realizadas en el Pacífico Sur, Brinkhuis et al. (2003a) y Bijl et al. (2013a) establecieron la PO de Enneadocysta dictyostila a los $45.5 \mathrm{Ma}$, registrándose en la porción media a superior de la Zona SPDZ10 (Luteciano medio, 46.2 Ma-45.2 Ma). Por lo tanto, este evento acota la edad de las secciones a Luteciano medio o más joven.

Hacia la parte media-alta de la Zona I se encuentra la POC de Enneadocysta dictyostila registrada a los 45,2 Ma (Luteciano medio) en la Plataforma de Tasmania, estableciendo la base de la Zona SPDZ 11 (45.2 Ma-44 Ma, Bijl et al., 2013a). A su vez, Enneadocysta dictyostila es una de las especies características de la Zona SPDZ 12 (Luteciano medio a Bartoniano temprano, 44.0 Ma-40.0 Ma) junto a Thalassiphora pelagica y Deflandrea antarctica (Bijl et al., 2013a). La UO de Hystrichosphaeridium truswelliae a los 38.8 Ma (Brinkhuis et al., 2003a) sugiere una edad no menor que Bartoniano tardío. Por lo tanto, la Zona I comprendería un rango de edad acotado entre el Luteciano medio y el Bartoniano tardío y permitiría su correlación con las zonas SPDZ11, SPDZ12 y base de la SPDZ13 (Bijl et al., 2013a).

Recientemente, Guerstein et al. (2014) realizaron un análisis palinológico en los niveles superiores de la Formación Man Aike, que aflora al sur del Lago Argentino (sudoeste de la cuenca Austral, $50^{\circ} 21^{\prime} 45^{\prime \prime} \mathrm{S}, 72^{\circ} 14^{\prime} 30^{\prime \prime} \mathrm{O}$ ) y reconocieron la presencia de la asociación endémica-antártica con un alto predominio de Enneadocysta dictyostila. Sobre la base de un análisis estadístico composicional, estos autores determinaron que las asociaciones de quistes de dinoflagelados de la Formación Man Aike presentan una alta correlación con las asociaciones de la parte inferior del miembro superior de la FRT, analizada tanto en los testigos de YCF como en los afloramientos sobre la Ruta Nacional 40 donde Enneadocysta dictyostila es también muy abundante. Las asociaciones de ambas formaciones fueron comparadas con las zonas de quistes de dinoflagelados del Océano Pacífico (Bijl et al., 2013a) y las edades obtenidas fueron complementadas con resultados de análisis de foraminíferos, nanofósiles calcáreos y edades isotópicas de ${ }^{87} \mathrm{Sr} r{ }^{86} \mathrm{Sr}$ (Malumián, 1990; Concheyro, 1991 y Casadío et al., 2009, respectivamente). De esta manera,

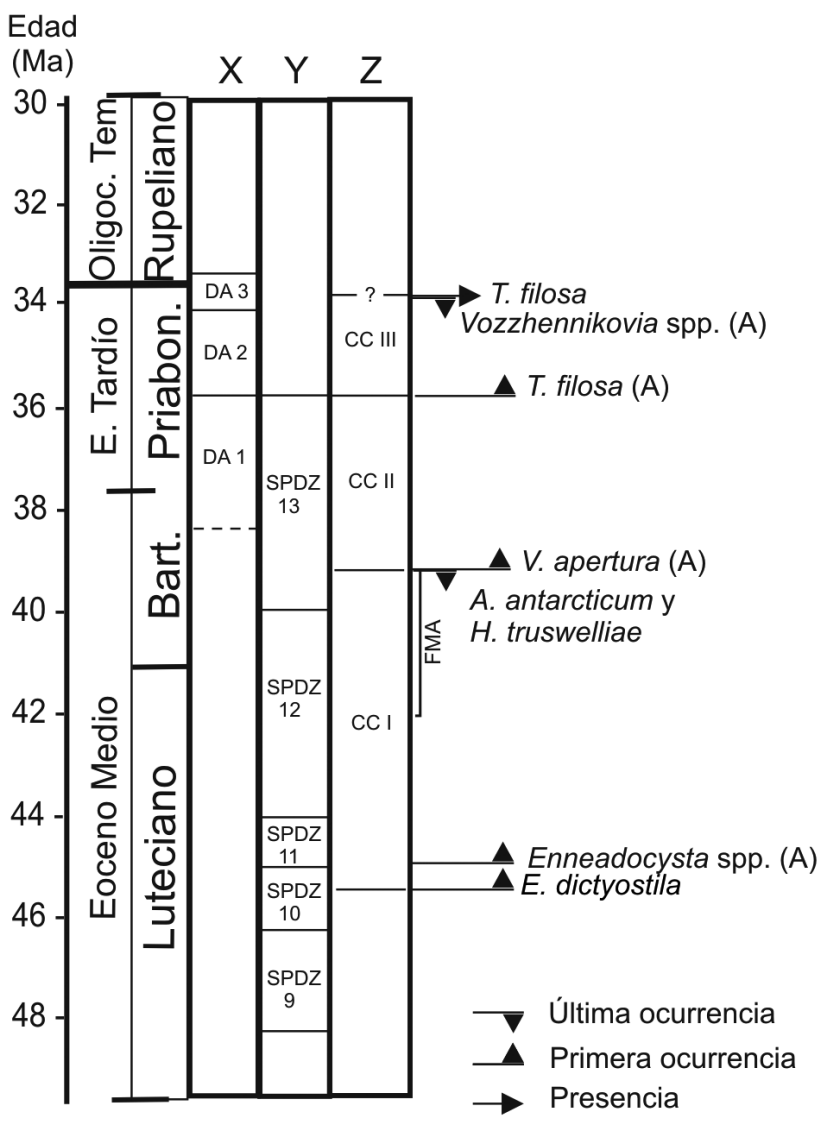

(A) Abundante

Figura 6. Eventos de quistes de dinoflagelados registrados en el miembro superior de la FRT expuesto en la localidad Ea. Cancha Carrera. Los rangos bioestratigráficos de las especies de dinoflagelados se toman de Brinkhuis et al. (2003b), Bijl et al. (2013a) y Sluijs et al. (2003), a excepción de la POC de V. apertura cuya disposición estratigráfica se basa en los resultados de este trabajo. $\mathbf{X}$, asociaciónes de quistes de dinoflagelados (Dinocyst association, DA) propuestas por Sluijs et al. (2003). Y, zonas de dinoflagelados de alta resolución del Pacífico Sur (South Pacific Dinocyst Zones, SPDZ) determinadas por Bijl, et al. (2013a). Z, zonas de quistes de dinoflagelados de la localidad Ea. Cancha Carrera (CC). FMA, parte superior de la Formación Man Aike.

Figure 6. Dinoflagellate cysts events recorded in the upper member of the FRT cropping out in Ea. Cancha Carrera. Biostratigraphic ranges of dinoflagellates taxa from Brinkhuis et al. (2003b), Bijl et al. (2013a) and Sluijs et al. (2003), except for the POC of V. aperura whose stratigraphic position is based on the results of this work. X, Dinocyst association (DA) proposed by Sluijs et al. (2003). Y, South Pacific Dinocyst Zones (SPDZ) determined by Bijl, et al. (2013a). Z, dinoflagellate cysts zones from Ea. Cancha Carrera (CC). FMA, upper part of Man Aike Formation.

Guerstein et al. (2014) determinaron para la parte superior de la Formación Man Aike y la parte inferior del miembro superior de la FRT una edad eocena media (Luteciano tardío a Bartoniano temprano) relacionada con las zonas de quistes de dinoflagelados SPDZ12 y SPDZ13 de Bijl et al. (2013a). La distribución de quistes de dinoflagelados de la FRT analizada en la Zona I de la sección Ea. Cancha Carrera (este trabajo) permite sugerir una edad equivalente a la establecida para la parte superior de la Formación Man Aike y la porción inferior 
del miembro superior de la FRT en cercanías de la localidad de Río Turbio.

La base de la Zona II puede definirse a los 38.8 Ma por la UO de Hystrichosphaeridium truswelliae (Brinkhuis et al., 2003a). Las asociaciones de quistes de dinoflagelados que conforman esta zona están integradas casi totalmente por Vozzhennikovia apertura. En los registros del Océano Pacífico Sur se reconocieron máximos de frecuencias de esta especie como parte de un complejo de Vozzhennikovia spp. entre los 50 Ma y 33.5 Ma (Bijl et al., 2013a). Según estos autores, su abundancia parece responder a factores ambientales sin que estos acmés puedan utilizarse como indicadores bioestratigráficos. Sin embargo, cabe mencionar que $V$. apertura es una especie característica de la Zona SPDZ13 (Bijl et al., 2013a) cuyo techo se registra a los 35.95 Ma. Teniendo en cuenta las asociaciones de quistes de dinoflagelados del límite Eoceno tardío-Oligoceno del Océano Pacífico Sur, Sluijs et al. (2003) propone una DA 1 compuesta por máximos de $V$. apertura, Spinidinium macmurdoense, Deflandrea antarctica y Phthanoperidinium spp. que se extiende desde el Bartoniano tardío hasta el Priaboniano, cuyo techo ( $\sim 35.5 \mathrm{Ma})$ es equivalente al techo de la Zona SPDZ13 de Bijl et al. (2013a). De esta manera, se interpreta que la Zona II definida en Ea. Cancha Carrea se extiende desde el Bartoniano tardío ( $\sim 38.8 \mathrm{Ma})$ al Priaboniano $(\sim 35.5 \mathrm{Ma})$ y puede correlacionarse con las zonas SPDZ13 y DA 1 de Bijl et al. (2013a) y Sluijs et al. (2003), respectivamente.

Hacia el techo de la sección, en la Zona III se observa un cambio en las asociaciones de quistes de dinoflagelados. La asociación endémica-antárctica se ve reemplazada por una comunidad con distribución cosmopolita representada por Turbiosphaera filosa, Protoperidinaceae, Imagidinium dispertitum, Operculodinium spp., Spiniferites spp. (principalmente $S$. pseudofurcatum) y Nematosphaeropsis sp. A. En trabajos realizados en el Océano Pacífico Sur, Sluijs et al. (2003), Stickley et al. (2004a,b) y Houben et al. (2013) observan un recambio durante el Eoceno tardío ( $\sim 35.5$ Ma) y lo relacionan con el comienzo de la profundización del conducto de Tasmania. Sluijs et al. (2003) define una DA 2 dominada por protoperidináceas cosmpolitas que se extiende desde los $\sim 35.5$ a los $34 \mathrm{Ma}$. La base de la DA 2 está determinada, entre otros eventos de dinoflagelados, por la POC de T. filosa a los 35.5 Ma (Sluijs et al., 2003). De esta manera, la PCO de T. filosa podría determinar la base de la Zona III a los $\sim 35.5 \mathrm{Ma}$.

Las UOs de Turbiosphaera filosa, Vozzhennikovia apertura y Spinidinium macmurdoense se reconocen a los 33.5 Ma (Brinkhuis et al., 2003a) y permiten definir una edad no más joven que Priaboniano para la sección analizada. Por lo tanto, la Zona III sugiere un rango de edad de $\sim 35.5$ a $33.5 \mathrm{Ma}$ y permitiría su correlación con la DA 2 de Sluijs et al. (2003).

En resumen, las zonas I-II muestran asociaciones de quistes de dinoflagelados con distribuciones endémicoantárticas, extendiéndose desde los $45.5 \mathrm{Ma}$ a los $\sim 35.5 \mathrm{Ma}$, las que se pueden correlacionar con las zonas SPDZ10-13 de Bijl et al. (2013a) y con la DA 1 de Sluij et al. (2003). La Zona III tendría una edad entre los 35.5 Ma y 33.5 Ma y muestra un aumento en las proporciones de quistes de dinoflagelados con distribución cosmopolita, lo que permite correlacionarla con la DA 2 de Sluij et al. (2003). De esta manera, se propone que el miembro superior de la FRT en la localidad Ea. Cancha Carrera se extiende desde el Luteciano medio hasta el final del Priaboniano.

\section{Paleoambiente}

Los dinoflagelados son altamente sensibles a pequeños cambios fisicoquímicos del agua superficial (e.g. temperatura, salinidad, concentración de nutrientes), así como a cambios en la profundidad y productividad. En reconstrucciones paleoecológicas basadas en quistes de dinoflagelados, la productividad del agua superficial se puede estimar usando la relación entre quistes peridinioideos (quistes - P) y gonyaulacoideos (quistes - G). Esta estimación se basa en considerar que los dinoflagelados generadores de quistes $-\mathrm{Pde}$ especies extintas habrían tenido el mismo hábito heterotrófico que el actual Protoperidinium, mientras que los quistes - G corresponderían a dinoflagelados con un comportamiento autotrófico o mixotrófico (Powell et al., 1992; Dale, 1996; Sluijs et al., 2005; Esper \& Zonneveld, 2007). Así, las altas frecuencias relativas de quistes peridinioideos podrían indicar una elevada concentración de nutrientes disueltos y aportar información sobre los niveles tróficos de las agua superficiales en el pasado (Goodman \& Ford, 1983; Wrenn \& Hart, 1988; Mao \& Mohr, 1995; Brinkhuis et al., 2003b; Sluijs et al., 2003; Bijl et al., 2010; entre otros). De esta manera, el análisis conjunto de la distribución de frecuencias de quistes de dinoflagelados y los resultados del estudio sedimentológico del miembro superior de la FRT permite reconstruir el paleoambiente y las condiciones del agua superficial durante el Eoceno medio a tardío en el área de estudio.

La Zona I se caracteriza por la alternancia de las máximas frecuencias de Enneadocysta dictyostila, Deflandrea spp. (principalmente $D$. antarctica) y Vozzhennikovia apertura. Röhl et al. (2004) registraron predominancias alternantes de Enneadocysta spp. y Deflandrea spp. en el Eoceno medio de la plataforma de Tasmania. Dichos autores correlacionaron las altas abundancias de Enneadocysta spp. (quistes - G) con importantes concentraciones de carbonato de calcio, mientras que los máximos de Deflandrea spp. (quistes - P) coinciden con los intervalos pobres en carbonato de calcio y ricos en nutrientes. Basándose en esta información, Röhl et al. (2004) propusieron que los máximos de Enneadocysta podrían reflejar aguas relativamente más cálidas, oligotróficas, en áreas de plataforma, mientras que los máximos porcentajes de Deflandrea indicarían aguas frías y condiciones eutróficas en ambientes someros. A su vez, en numerosos trabajos se relaciona a $D$. antarctica con $V$. apertura (quistes $-\mathrm{P}$ ) y se considera a ambas especies típicas de aguas circum-antárticas frías, poco profundas y con altos niveles tróficos (Wilson, 1967; Hannah, 1997; Mohr, 1990; Brinkhuis et al., 2003a; MacPhail \& Truswell, 2004; Sluijs et al., 2003, 2005; Röhl et al., 2004; Warnaar et al., 2009; Bijl et al., 2011; González Estebenet et al., 2014b). Los episodios de profundización se pueden relacionar con altas frecuencias de E. dictyostila, 
mientras que los episodios de somerización se pueden asociar al predominio de Deflandrea spp. (Pross \& Brinkhuis, 2005). De esta manera, considerando la alternancia en el predominio de E. dictyostila versus Deflandrea spp. y/o V. apertura se reconocen en esta zona dos ascensos relativos del nivel del mar.

Las asociaciones de la Zona II muestran el predominio de Vozzhennikovia apertura, especie característica de aguas frías y someras con alta disponibilidad de nutrientes. González Estebenet et al. (2014b) estudiaron las asociaciones de quistes de dinoflagelados del miembro superior de la FRT en perfiles aflorantes sobre la Ruta Nacional 40 y determinaron dos zonas con un importante predominio de esta especie. En dichas secciones $V$. apertura predomina en los niveles que fueron interpretados como depositados en un ambiente estuárico por Rodríguez Raising et al. (2014) influenciado por sistemas de descargas fluviales. Los aportes continentales habrían generado un aumento en la disponibilidad de nutrientes y alterado las concentraciones de salinidad generando condiciones de estrés ambiental. Estas condiciones se manifiestan en los caracteres tafonómicos de un arrecife de ostras desarrollado en niveles equivalentes aflorantes $\sim 50$ $\mathrm{km}$ al sur de la sección aquí estudiada (Rodriguez Raising et al., 2014). De acuerdo con estas evidencias González Estebenet et al. (2014b) propusieron que $V$. apertura sería el quiste de un dinoflagelado tolerante a condiciones de estrés ambiental. El predominio casi exclusivo de $V$. apertura en la Zona II del perfil expuesto en Ea. Cancha Carrera permite inferir un ambiente vinculado con áreas costeras sujeto a descargas fluviales.

En la Zona III se observa el recambio de especies endémicas (Zona II) por taxa cosmopolitas. La parte inferior de esta zona (muestra CC 11) se encuentra dominada por Turbiosphaera filosa, donde se evidencia una marcada variación en la forma, estructura y grado de unión de los procesos. Archangelsky (1968) en la sección superior de la FRT (testigo YCF/ D-15) registró el polimorfismo en T. filosa destacando distintos grados en el desarrollo morfológico de los quistes, siendo el estado más avanzado el que presenta procesos claramente definidos. Pross (2001) y Pross \& Schmiedl (2002) reconocieron una situación similar para Thalassiphora pelagica, una especie perteneciente a la misma subfamilia (Cribroperidinioidea), en estudios realizados en el Oligoceno del sudoeste de Alemania. Dichos autores propusieron que $T$. pelagica habría tenido un ciclo de vida holoplanctónico con diferentes grados de desarrollo morfológico e interpretaron que la fase más evolucionada presenta un quiste con perifragma bien expandido. Según dichos autores, el predominio de quistes con un desarrollo temprano o incompleto se correlaciona con episodios de máximos aportes fluviales, los que provocarían un aumento en la productividad y una reducción en la salinidad en las aguas superficiales como así también una menor disponibilidad de oxígeno en la columna de agua más profunda. Para la parte superior de la FRT Rodriguez Raising (2010) sugirió un ambiente de depositación influenciada por descargas fluviales. Si bien se desconocen las causas de los distintos grados de desarrollo de T. filosa, es probable que la variabilidad de los procesos responda a factores ambientales semejantes a los que Pross (2001) y Pross \& Schmiedl (2002) propusieron para T. pelagica. Asimismo, la muestra de niveles inmediatamente supradyacentes (CC 12), presenta palinomorfos no marinos en concordancia con el ambiente sugerido a partir del análisis de estratigrafía secuencial (Rodriguez Raising, 2010).

Hacia el techo de la sección (muestras CC 13 y CC 14), se registra una disminución en las especies endémicas y un aumento en la riqueza específica y abundancia de taxa cosmopolitas. Entre ellas, se destacan las mayores proporciones de Protoperidinaceae, las cuales se asocian a aguas superficiales ricas en nutrientes (Pross \& Brinkhuis, 2005; Zonneveld et al. 2010), mientras que los peridinioideos endémicos se encuentran considerablemente reducidos. Entre los gonyaulacoideos se reconocen especies típicas de ambientes neríticos externos a oceánicos de los géneros Nematosphaeropsis e Impagidinium (Dale, 1996; Marret \& Zonneveld 2003; Zonneveld et al., 2013) junto con Operculodinium spp. y Lingulodinium sp. con una distribución en ambientes nerítico interno a externo (Pross \& Brinkhuis, 2005). Los ejemplares de Turbiosphaera filosa de estos niveles, en contraposición a lo observado en la muestra CC 11 , se caracterizan por procesos bien desarrollados y son muy pocos los ejemplares que presentan un desarrollo incipiente. Retomando la analogía con Thalassiphora pelagica y considerando que T. filos $a$ pudo haber respondido de manera similar, en condiciones marinas normales (no afectadas por descargas fluviales y aguas ricas en nutrientes), estas especies forman parte de asociaciones diversas y sólo se reconoce el estadio de mayor desarrollo. De esta manera, la parte cuspidal de la sección podría haberse depositado en áreas de plataforma externa sujeta a procesos de surgencia de agua de fondo (upwelling) responsables de altas concentraciones de nutrientes en las aguas superficiales. De manera análoga, sobre la Plataforma Continental Argentina se reconocen actualmente procesos de afloramiento de aguas profundas a través de un mecanismo de surgencia acompañado del ingreso hacia el interior de la plataforma de aguas subsuperficiales de la Corriente de Malvinas. Este mecanismo ha sido corroborado utilizando modelos numéricos de circulación oceánica del Atlántico Sudoccidental (Matano \& Palma, 2008; Palma et al., 2008; Combes \& Matano, 2014) y es consistente con los análisis de observaciones hidrográficas realizadas sobre la Plataforma Patagónica por Piola et al. (2010).

\section{Paleocirculación oceánica en áreas circum-antárticas}

Es importante notar que entre la Zona II y la Zona III se observa un reemplazo de especies endémicas-antárticas (Vozzhennikovia apertura y Deflandrea antarctica) por taxa con distribución cosmopolita (Turbiosphaera filosa, Operculodinium spp. y protoperidiniáceas). La abundancia de la asociación endémica con máximas frecuencias de Enneadocysta dictyostila, $D$. antarctica y $V$. apertura se registra en varias unidades eocenas del Hemisferio Sur (Archangelsky 1968, 1969; Wrenn \& Beckmann, 1982; Wrenn \& Hart, 1988; Cocozza \& Clarke, 1992; Mao \& Morh, 1995; Brinkhuis et al., 2003a,b; Sluijs et al., 2003; Sticley 
et al., 2004a; Guerstein et al., 2008; Bijl et al., 2011, 2013a; Guerstein et al., 2014, Douglas et al., 2014, entre otros). Su expansión parece haber estado estrechamente relacionada con un esquema de circulación oceánica con amplios giros subpolares en sentido horario alrededor de Antártida (Huber et al., 2004; Figura 7). Según estos autores, dichos giros habrían generado un mecanismo selectivo intrínseco, denominado "trampa refrigeradora" ("cold trap"), responsable del endemismo de especies tolerantes a temperaturas del agua superficial relativamente bajas. Este patrón de circulación oceánica fue verificado teniendo en cuenta la distribución de los quistes de dinoflagelados de los testigos ODP 189 al Este y al Oeste de Tasmania (Huber et al., 2004; Stickley et al., 2004a,b). Posteriormente, Guerstein \& Daners (2010), teniendo en cuenta los resultados de los experimentos de Huber et al. (2004), consideraron que el mecanismo propuesto

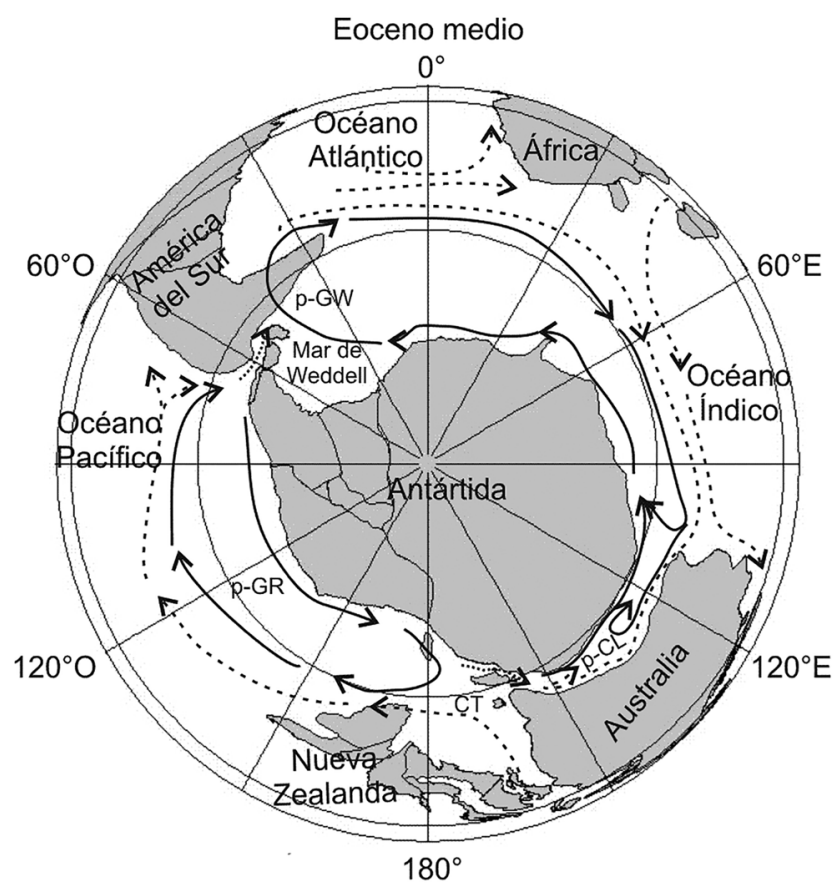

Figura 7. Mapa paleogeográfico del Eoceno medio a partir de Plate Tectonic Reconstructions On-line Paleogeographic Mapper (Schettino \& Scotese, 2005). Áreas en gris representan corteza continental. Flechas enteras indican los esquemas de circulación oceánica con giros subpolares y aguas relativamente frías. Flechas con líneas cortadas representan corrientes cálidas (modificado a partir de Huber et al., 2004; Guerstein \& Daners, 2010). Flechas punteadas señalan un flujo de aguas someras a través de los conductos oceánicos (Livermore et al., 2007; Eagles \& Jokat, 2014; Bijl et al., 2013b; Sijp et al., 2014). Abreviaciones: p-GW, proto-Giro de Weddell; p-GR, proto-Giro de Ross; CT, Corriente de Tasmania; $\mathbf{p - C L}$, proto-Corriente de Leeuwin.

Figure 7. Middle Eocene paleogeographic map derived from Plate Tectonic Reconstructions On-line Paleogeographic Mapper (Schettino \& Scotese, 2005). Gray areas indicate continental crust. Arrows with black lines indicate schematic oceanic circulation subpolar gyres and relatively cold waters. Arrows with dashed lines represent warm currents (modified from Huber et al., 2004; Guerstein \& Daners, 2010). Dotted arrows indicate shallow water flows through ocean gateways (modified after Huber et al., 2004 and Guerstein \& Daners, 2010). Abbreviations: p-WG, proto-Weddell Gyre; p-RG, proto-Ross Gyre; TC, Tasman Current; $\mathbf{p}$-LC, proto-Leeuwin Current. para el Pacífico Sur podría haber funcionado igualmente para el Atlántico Sur y sugirieron la existencia de un giro en sentido horario, equivalente a un proto-Giro de Weddell (Figura 7).

Durante el Eoceno tardío-Oligoceno temprano tuvo lugar el reemplazo de especies endémicas por taxa cosmopolitas, principalmente de la familia Protoperidiniaceae (Mohr 1990; Brinkhuis et al., 2003a; Huber et al., 2004; Sluijs et al., 2003; Sticley et al., 2004a; Houben et al., 2011, 2013). La extinción de la asociación endémica parece estar forzada por la apertura y la profundización del Pasaje de Drake y el Conducto de Tasmania (Suijs et al., 2003; Stickley et al., 2004a; Guerstein et al., 2008a; Houben et al., 2011, 2013). Tales cambios paleogeográficos habrían generado un flujo de agua circumpolar irrestricto (Corriente Circumpolar Antártica incipiente) alterando la circulación oceánica de giros subpolares y las condiciones ambientales que favorecían el endemismo (Suijs et al., 2003; Stickley et al., 2004a; Huber et al., 2004; Guerstein et al., 2008a). Es posible que en la parte superior de la sección estudiada (Zona III) el predominio de Turbiosphaera filosa junto con el aumento de Protoperidiniaceae y otras especies cosmopolitas, en reemplazo de las especies endémicas, reflejen para el área del Pasaje de Drake el inicio de los cambios en los patrones de circulación oceánica reconocidos globalmente a partir del Oligoceno.

\section{CONCLUSIONES}

Los eventos de quistes de dinoflagelados registrados en el miembro superior de la FRT se compararon con los publicados en el Océano Pacífico Sur por Brinkhuis et al. (2003), Sluijs et al. (2003), Sticley et al. (2004a) y Bijl et al. (2013a). Las asociaciones de quistes de dinoflagelados reconocidas en el sudoeste de la Patagonia corresponden al intervalo estratigráfico comprendido entre la Zona SPDZ 10 y la Zona SPDZ 13 de Bijl et al. (2013a) y las zonas de quistes de dinoflagelados DA 1 y DA 2 de Sluijs et al. (2003). Esta correlación permite establecer un rango de edad para el miembro superior de la FRT que varía entre los 45.5 Ma (Luteciano medio) y los $33.5 \mathrm{Ma}$ (Priaboniano terminal).

Se reconocieron tres zonas de quistes de dinoflagelados. Las frecuencias alternantes entre Enneadocysta dictyostila, Deflandrea antarctica y Vozzhennikovia apertura en la Zona I sugieren fluctuaciones del nivel del mar. Los episodios de profundización se relacionan con altas frecuencias de $E$. dictyostila, mientras que los episodios de somerización se asocian al predominio de $D$. antarctica y/o $V$. apertura. El predominio casi exclusivo de $V$. apertura en la Zona II permite inferir un ambiente vinculado con áreas costeras sujeto a descargas fluviales. En la base de la Zona III la abundancia y el marcado polimorfismo de Turbiosphaera filosa podrían responder a oscilaciones en las concentraciones de salinidad y oxígeno en la columna de agua en el área de depositación. Hacia la parte alta de la sección, la asociación compuesta por Protoperidinaceae, Nematosphaeropsis, Impagidinium, Operculodinium spp. y Turbiosphaera filosa con procesos bien desarrollados, sugiere un ambiente de plataforma externa posiblemente influenciada por sistemas de surgencia de agua de fondo. 
Las zonas I y II se encuentran altamente dominadas por especies típicas de la asociación endémica-antártica (Enneadocysta dictyostila, Deflandrea antarctica y Vozzhennikovia apertura), mientras que en la Zona III predominan especies cosmopolitas (Tubiosphaera filosa, protoperidináceas y Operculodinium spp., entre otras). El reemplazo de las especies endémicas por taxa cosmopolitas podría señalar el inicio de los cambios en los patrones de circulación oceánica globales reconocidos en la transición Eoceno-Oligoceno.

\section{AGRADECIMIENTOS}

Los autores agradecen a A.M. Ribeiro, editora de la Revista Brasileira de Paleontología y a dos revisores anónimos por sus oportunas recomendaciones; a P.A. de Souza por su significativa colaboración en la traducción del resumen al Portugués y revisión del abstract; a M.R. Raising por ceder las muestras para el estudio palinológico aquí realizado y a O. Cárdenas por las extracción fisicoquímica de las mismas. Este trabajo fue financiado con proyectos de la Agencia Nacional de Promoción Científica y Tecnológica (PICT 89/09), del Consejo Nacional de Investigaciones Científicas y Técnicas (PIP: 112-201101-00566) y de la Universidad Nacional del Sur (PGI 24/H125).

\section{REFERENCIAS}

Archangelsky, S. 1968. Sobre el paleomicroplancton del Terciario inferior de Río Turbio, provincial de Santa Cruz. Ameghiniana, 5:406-416.

Archangelsky, S. 1969. Estudio del paleomicroplancton de la Formación Río Turbio (Eoceno), Provincia de Santa Cruz. Ameghiniana, 6:181-218.

Arguijo, M.H. \& Romero, E.J. 1981. Análisis bioestratigráfico de formaciones portadoras de tafofloras terciarias. In: CONGRESO GEOLÓGICO ARGENTINO, 8, 1981. Actas, Buenos Aires, AGA, p. 691-717.

Biddle, K.; Uliana, M.; Mitchum, R.; Fitzgerald, M. \& Wright, R. 1986. The stratigraphic and structural evolution of the central and eastern Magallanes Basin, southern South America. In: P.A. Allen \& P. Homewood (eds.) Foreland Basins, Gent, International Association of Sedimentologists, p. 41-61 (Special Publication 8). doi:10.1002/9781444303810.ch2

Bijl, P.K.; Bendle, J.A.P; Bohaty, S.M.; Pross, J.; Schouten, S.; Tauxe, L.; Stickley, C.E.; McKay, R.M.; Röhl, U.; Olney, M.; Sluijs, A.; Escutia, C.; Brinkhuis, H. \& Expedition 318 Scientists. 2013b. Eocene cooling linked to early flow across the Tasmanian Gateway. Proceedings of the National Academy of Sciences, 110:9645-9650. doi:10.1073/pnas. 1220872110

Bijl, P.K.; Houben, A.J.; Schouten, S.; Bohaty, S.M.; Sluijs, A.; Reichart, G.; Damsté J.S. \& Henk, B.H. 2010. Transient Middle Eocene atmospheric $\mathrm{CO}_{2}$ and temperature variations. Science, 330:819-821. doi:10.1126/science. 1193654

Bijl, P.K.; Pross, J.; Warnaar, J.; Stickley, C.E.; Huber, M.; Guerstein, R.; Houben, A.J.P.; Sluijs, A.; Visscher, H. \& Brinkhuis, H. 2011. Environmental forcings of Paleogene Southern Ocean dinoflagellate biogeography. Paleoceanography, 26:PA1202. doi: $10.1029 / 2009 P A 001905$
Bijl, P.K.; Sluijs, A. \& Brinkhuis, H. 2013a. A magneto-and chemostratigraphically calibrated dinoflagellate cyst zonation of the early Palaeogene South Pacific Ocean. Earth-Science Reviews, 124:1-31. doi:10.1016/j.earscirev.2013.04.010

Bohaty, S.M \& Zachos, J.C. 2003. Significant Southern Ocean warming event in the late middle Eocene. Geology, 31:10171020. doi:10.1130/G19800.1

Bohaty, S.M.; Zachos, J.C.; Florindo, F. \& Delaney, M.L. 2009. Coupled greenhouse warming and deep-sea acidification in the middle Eocene. Paleoceanography, 24:PA2207. doi:10.1029/2008PA001676

Brinkhuis, H.; Bujak, J.P.; Smit, J.; Versteegh, G.J.M. \& Visscher, H. 1998. Dinoflagellate-based sea surface temperature reconstructions across the Cretaceous-Tertiary boundary. Palaeogeography, Palaeoclimatology, Palaeoecology, 141:6783. doi:10.1016/S0031-0182(98)00004-2

Brinkhuis, H.; Munsterman, D.M.; Sengers, S.; Sluijs, A.; Wanaar, J. \& Williams, G.L. 2003a. Late Eocene to Quaternary dinoflagellate cysts from ODP Site 1168, off western Tasmania. Proceedings of the Ocean Drilling Program, 189:1-36.

Brinkhuis, H.; Sengers, S.; Sluijs, A.; Warnaar, J. \& Williams, G.L. 2003b. Latest Cretaceous to earliest Oligocene, and Quaternary dinoflagellate cysts from ODP Site 1172, East Tasman Plateau. Proceedings of the Ocean Drilling Program, 189:1-48.

Calegari, R.; Baldi, M.J. \& Pioli, O. 1993. Sismoestratigrafía del Terciario de cuenca Austral. Aplicación en proyectos exploratorios. Boletín de Informaciones Petroleras, 10:2-23.

Casadío, S.; Griffin, M.; Marenssi, S.; Net, L.; Parras, A.; Raising, M.R. \& Santillana, S. 2009. Paleontology and sedimentology of Middle Eocene rocks in Lago Argentino area, Santa Cruz Province, Argentina. Ameghiniana, 46:27-47.

Cocozza, C.D. \& Clarke, C.M. 1992. Eocene microplankton from $\mathrm{La}$ Meseta Formation, northern Seymour Island. Antarctic Science, 4:355-362. doi:10.1017/S0954102092000506

Combes, V. \& Matano, R.P. 2014. A two-way nested simulation of the oceanic circulation in the Southwestern Atlantic. Journal of Geophysical Research, 119:731-756. doi:10.1002/2013JC009498

Concheyro, A. 1991. Nanofósiles calcáreos de la Formación Man Aike (Eoceno, sudeste del Lago Cardiel) Santa Cruz, Argentina. Ameghiniana, 28:385-399.

Crouch, E.M.; Dickens, G.R.; Brinkhuis, H.; Aubry, M.-P.; Hollis, C.J.; Rogers, K.M. \& Visscher, H. 2003. The Apectodinium acme and terrestrial discharge during the Paleocene- Eocene Thermal Maximum: new palynological, geochemical and calcareous nannoplankton observations at Tawanui, New Zealand. Palaeogeography, Palaeoclimatology, Palaeoecology, 194:387-403. doi:10.1016/S0031-0182(03)00334-1

Dale, B. 1996. Dinoflagellate cyst ecology: modelling and geological applications. In: J. Jansonius \& D.C. McGregor (eds.) Palynology: principles and applications, The American Association of Stratigraphic Palynologists Foundation, Publishers Press, p. 1249-1276.

Douglas, P.M.; Affek, H.P.; Ivany, L.C.; Houben, A.J.; Sijp, W.P.; Sluijs, A.; Schouten, S. \& Pagani, M. 2014. Pronounced zonal heterogeneity in Eocene southern high-latitude sea surface temperatures. Proceedings of the National Academy of Sciences, 111:6582-6587. doi:10.1073/pnas.1321441111

Eagles, G. \& Jokat, W. 2014. Tectonic reconstructions for paleobathymetry in Drake Passage. Tectonophysics, 611:28-50. doi:10.1016/j.tecto.2013.11.021

Esper, O. \& Zonneveld, K.A.F. 2007. The potential of organic-walled dinoflagellate cysts for the reconstruction of past sea-surface 
conditions in the Southern Ocean. Marine Micropaleontology, 65:185-212. doi:10.1016/j.marmicro.2007.07.002

Fensome, R.A.; MacRae, R.A. \& Williams, G.L. 2008. DINOFLAJ2, Version 1. Dallas, American Association of Stratigraphic Palynologists, Data Series 1.

González Estebenet, M.S.; Guerstein, G.R. \& Alperin, M.I. 2014a. Dinoflagellate cyst distribution during the middle Eocene in the Drake Passage area: paleoceanographic implications. Ameghiniana, 51:500-509. doi: 10.5710/ AMGH.06.08.2014.2727

González Estebenet, M.S.; Guerstein, G.R. \&Raising, M.E.R. 2014b. Middle Eocene Dinoflagellate cysts from Santa Cruz Province, Argentina: biostratigraphy and paleoenvironment. Review of Palaeobotany and Palynology, 211:55-65. doi:10.1016/j. revpalbo.2014.09.002

Goodman, D.K. \& Ford, L.N. 1983. Preliminary dinoflagellate biostratigraphy for the middle Eocene to lower Oligocene from the southwest Atlantic Ocean. In: W.J. Ludwig; V.A. Krasheninnikov \& S.W. Wise Jr. (eds.) Initial Reports of the Deep Sea Drilling Project Press, Washington, p. 859-877. doi:10.2973/ dsdp.proc. 71.131 .1983

Guerstein, G.R.; Brinkhuis, H. \& Daners, G. 2008a. Eocene circulation and dinoflagellate distribution in the Southwestern Atlanctic Ocean. In: REUNIÓN ARGENTINA DE SEDIMENTOLOGÍA, 12, 2008. Actas, Buenos Aires, AAS, p. 81.

Guerstein, G.R. \& Daners, G. 2010. Distribución de Enneadocysta (Dinoflagellata) en el Paleógeno del Atlántico Sudoccidental: implicancias paleoceanográficas. Ameghiniana, 47:461-478. doi:10.5710/AMGH.v47i4.5

Guerstein, G.R.; Guler, M.V.; Williams, G.L.; Fensome, R.A. \& Chiesa, J.O. 2008b. Mid Palaeogene dinoflagellate cysts from Tierra del Fuego, Argentina: biostratigraphy and palaeoenvironments. Journal of Micropalaeontology, 27:75-94. doi:10.1144/jm.27.1.75

Guerstein, G.R.; González, M.S.E.; Alperin, M.I.; Casadío, S.A. \& Archangelsky, S. 2014. Correlation and paleoenvironments of middle Paleogene marine beds based on dinoflagellate cysts in southwestern Patagonia, Argentina. Journal of South American Earth Sciences, 52:166-178. doi:10.1016/j.jsames.2014.02.011

Guerstein, G.R.; Raising, M.E.R.; Casadío; S.; Marenssi, S. \& Cárdenas, O. 2010. Palinología del Miembro Inferior de la Formación Río Turbio (Eoceno inferior a medio) en el cañón del río Guillermo, suroeste de Santa Cruz, Argentina. In: CONGRESO ARGENTINO DE PALEONTOLOGÍA Y BIOESTRATIGRAFÍA, 10, 2010. Resúmenes, La Plata, APA, R93.

Hammer, Ø.; Harper, D.A.T. \& Ryan, P.D. 2001. PAST: Paleontological Statistics Software Package for Education and Data Analysis. Palaeontología Electrónica, 4:9. Avaliable at < http://palaeo-electronica.org/2001_1/past/issue1_01.htm>; accessed on 21/11/2015.

Hannah, M.J. 1997. Climate controlled dinoflagellate distribution in late Eocene-earliest Oligocene strata from CIROS-01 drillhole, McMurdo Sound, Antarctica. Terra Antartica, 4:73-78.

Houben, A.J.P.; Bijl, P.K.; Guerstein, R.G.; Sluijs, A. \& Brinkhuis, H. 2011. Malvinia escutiana, a new biostratigraphically important Oligocene dinoflagellate cyst from the Southern Ocean. Review of Palaeobotany and Palynology, 165:3-4. doi:10.1016/j. revpalbo.2011.03.002

Houben, A.J. et al. 2013. Reorganization of Southern Ocean plankton ecosystem at the onset of Antarctic glaciations. Science, 340:341344. doi:10.1126/science. 1223646
Huber, M.; Brinkhuis, H.; Stickley, C.E.; Döös, K.; Sluijs A.; Warnaar, J.; Schellenberg, S.A. \& Williams, G.L. 2004. Eocene circulation of the Southern Ocean: Was Antarctica kept warm by subtropical waters? Paleoceanography, 19:PA4026. doi:10.1029/2004PA001014

Kraemer, P.E.; Ploszkiewicz, J.V. \& Ramos, V.A. 2002. Estructura de la Cordillera Patagónica austral entre los $46^{\circ}$ y los $52^{\circ} \mathrm{S}$. In: CONGRESO GEOLÓGICO ARGENTINO, 15, 2002. Actas, Buenos Aires, AGA, p. 353-364.

Lagabrielle, Y.; Goddéris, Y.; Donnadieu, Y.; Malavieille, J. \& Suarez, M. 2009. The tectonic history of Drake Passage and its possible impacts on global climate. Earth and Planetary Science Letters, 279:197-211. doi:10.1016/j.epsl.2008.12.037

Livermore, R.; Hillenbrand, C.D.; Meredith, M. \& Eagles, G. 2007. Drake Passage and Cenozoic climate: an open and shut case? Geochemistry, Geophysics, Geosystems, 8:1. doi:10.1029/2005GC001224

MacPhail, M.K. \& Truswell, E.M. 2004. Palynology of Site 1166, Prydz Bay, East Antarctica. Proceedings of the Ocean Drilling Program, 188:1-43.

Malumián, N. 1990. Foraminíferos de la Formación Man Aike (Eoceno, Sureste Lago Cardiel) Provincia de Santa Cruz. Revista de la Asociación Geológica Argentina, 45:365-385.

Malumián, N. 2002. El Terciario marino. Sus relaciones con el eustatismo. In: CONGRESO GEOLÓGICO ARGENTINO, 15, 2002. Relatorio, Buenos Aires, AGA, p. 237-244.

Malumián, N. \& Náñez, C. 2002. Los foraminíferos de la provincia de Santa Cruz. Su significado geológico. In: CONGRESO GEOLÓGICO ARGENTINO, 15, 2002. Relatorio, Buenos Aires, AGA, p. 481-494.

Malumian, N.; Panza, J.L.; Parisi, C.; Nanez, C.; Carames, A. \& Torre, E. 2000. Hoja Geologica 5172-III-Yacimiento Rio Turbio, provincia Santa Cruz, 1:250.000. Buenos Aires, Servicio Geológico Minero Argentino, 108 p. (Boletín 247).

Marret, F. \& Zonneveld, K.A. 2003. Atlas of modern organic-walled dinoflagellate cyst distribution. Review of Palaeobotany and Palynology, 125:1-200. doi:10.1016/S0034-6667(02)00229-4

Mao, S. \& Mohr, B.A.R. 1995. Middle Eocene dinocysts from Bruce Bank (Scotia Sea, Antarctica) and their paleoenvironmental and paleogeographic implications. Review of Palaeobotany and Palynology, 86:235-263. doi:10.1016/0034-6667(94)00138-A

Matano, R.P. \& Palma E.D. 2008. On the upwellling of downwelling currents. Journal of Physical Oceanography, 113:2482-2500. doi:10.1175/2008JPO3783.1

Mohr, B.A.R. 1990. Eocene and Oligocene sporomorphs and dinoflagellate cysts from Leg 113 drill sites, Weddell Sea, Antarctica. Proceedings of the Ocean Drilling Program, 113:595-612. doi:10.2973/odp.proc.sr.113.140.1990

Nullo, F.E.; Panza, J.L. \& Blasco, G. 1999. Jurásico y Cretácico de la cuenca Austral. In: R. Caminos (ed.) Geología Argentina, Buenos Aires, Instituto de Geología y Recursos Minerales, SEGEMAR, p. 399-416 (Anales 29).

Olivero, E.B. \& Malumián, N. 1999. Eocene stratigraphy of southeastern Tierra del Fuego island, Argentina. American Association of Petroleum Geologists Bulletin, 83:295-313.

Olivero, E.B. \& Malumián, N. 2008. Mesozoic-Cenozoic stratigraphy of the Fueguian Andes, Argentina. Geológica Acta, 6:5-18.

Palma, E.D.; Matano, R.P. \& Piola, A.R. 2008. A numerical study of the Southwestern Atlantic Shelf circulation: stratified ocean response to local and offshore forcing. Journal of Geophysical Research, 113:C11010. doi:10.1029/2007JC004720 
Piola, A.R.; Martinez Avellaneda, N.; Guerrero, R.A.; Jardon, F.P.; Palma, E.D. \& Romero, S.I. 2010. Malvinas-slope water intrusions on the northern Patagonia continental shelf. Ocean Sciences, 6:345-359.

Powell, A.J.; Lewis, J. \& Dodge, J.D. 1992. The palynological expressions of post-Paleogene upwelling: a review. In: C.P. Summerhayes; W.L. Prell \& K.C. Emeis (eds.) Upwelling systems: evolution since the Early Miocene, London, The Geological Society, p. 215-226 (Special Publication 64).

Pross, J. 2001. Paleo-oxygenation in Tertiary epeiric seas: evidence from dinoflagellate cysts. Palaeogeography, Palaeoclimatology, Palaeoecology, 166:369-381. doi:10.1016/ S0031-0182(00)00219-4

Pross, J. \& Brinkhuis, H. 2005. Organic-walled dinoflagellate cysts as paleoenvironmental indicators in the Paleogene; a synopsis of concepts. Paläontologische Zeitschrift, 79:53-59. doi:10.1007/ BF03021753

Pross, J. \& Schmiedl, G. 2002. Early Oligocene dinoflagellate cysts from the Upper Rhine Graben (SW Germany): paleoenvironmental and paleoclimatic implications. Marine Micropaleontology, 45:1-24. doi:10.1016/S0377-8398(01)00046-9

Ramos, V.A. 2005. Seismic ridge subduction and topography: foreland deformation in the Patagonian Andes. Tectonophysics, 399:73-86. doi:10.1016/j.tecto.2004.12.016

Rodríguez Raising, M.E. 2010. Estratigrafía secuencial de los depósitos marinos y continentales del Eoceno - Oligoceno temprano de la cuenca Austral, sudoeste de la provincia de Santa Cruz. Programa de Post-grado en Geología, Universidad Nacional del Sur, Argentina, Tesis doctoral, 203 p.

Rodríguez Raising, M.; Casadío, S.; Pearson, N.; Mangano, G.; Buatois, L. \& Griffin, M. 2014. Paleoenvironmental setting and description of an estuarine oyster reef in the Eocene of Patagonia, southern Argentina. Journal of South American Earth Sciences, 56:242-250. doi:10.1016/j.jsames.2014.09.009

Röhl, U.; Brinkhuis, H.; Stickley, C.E.; Fuller, M.; Schellenberg, S.A.; Wefer, G. \& Williams, G.L. 2004. Sea level and astronomically induced environmental changes in middle and late Eocene sediments from the East Tasman Plateau. In: N.F. Exon; M. Malone \& J.P. Kennett (eds.) Climate evolution of the Southern Ocean and Australia's northward flight from Antarctica, Washington, American Geophysical Union, p. 127-151 (Geophysical Monograph Series 151). doi:10.1029/151GM09

Scher, H.D. \& Martin, E.E. 2006. Timing and climatic consequences of the opening of Drake passage. Science, 312:428-430. doi:10.1126/science. 1120044

Schettino, A. \& Scotese, C.R. 2005. Apparent polar wander paths for the major continents ( $200 \mathrm{Ma}$ to present day): a paleomagnetic reference frame for global plate tectonic reconstructions. Geophysical Journal International, 163:727-759. doi:10.1111/ j.1365-246X.2005.02638.x

Sijp, W.P.; Anna, S.; Dijkstra, H.A.; Flögel, S.; Douglas, P.M. \& Bijl, P.K. 2014. The role of ocean gateways on cooling climate on long time scales. Global and Planetary Change, 119:1-22. doi:10.1016/j.gloplacha.2014.04.004

Sluijs, A.; Brinkhuis, H.; Stickley, C.E.; Warnaar, J.; Williams, G.L. \& Fuller, M. 2003. Dinoflagellate cysts from the Eocene / Oligocene transition in the Southern Ocean; results from ODP Leg 189. Proceedings of the Ocean Drilling Program, 189:1-42.
Sluijs, A.; Brinkhuis, H.; Williams, G.L. \& Fensome, R.A. 2009. Taxonomic revision of some Cretaceous-Cenozoic spiny organic-walled peridiniacean dinoflagellate cysts. Review of Palaeobotany and Palynology, 154:34-53. doi:v10.1016/j. revpalbo.2008.11.006

Sluijs, A.; Pross, J. \& Brinkhuis, H. 2005. From greenhouse to icehouse; organic-walled dinoflagellate cysts as paleoenvironmental indicators in the Paleogene. Earth Science Reviews, 68:281-315. doi:10.1016/j.earscirev.2004.06.001

Stickley, C.E. Brinkhuis, H.; McGonigal, K.L.; Chaproniere, G.C.H.; Fuller, M.; Kelly, D.C.; Nürnberg, D.; Pfuhl, H.A.; Schellenberg, S.A.; Schoenfeld, J.; Suzuki, N.; Touchard, Y.; Wei, W.; Williams, G.L.; Lara, J. \& Stant, S.A. 2004b. Late Cretaceous-Quaternary biomagnetostratigraphy of ODP Sites 1168, 1170, 1171, and 1172, Tasmanian Gateway. Proceedings of the Ocean Drilling Program, 189:1-57.

Stickley, C.E.; Brinkhuis, H.; Schellenberg, S.A.; Sluijs, A.; Röhl, U.; Fuller, M.; Grauert, M.; Huber, M.; Warnaar, J. \& Williams, G.L. 2004a. Timing and nature of the deepening of the Tasmanian gateway. Paleoceanography, 19:PA4027. doi:10.1029/2004PA001022

Vandenberghe, N.; Speijer, R.P. \& Hilgen, F.J. 2012. The Paleogene period. In: F.M. Gradstein; J.G. Ogg; M. Schmitz \& G. Ogg (eds.) The Geologic Time Scale 2012, Elsevier, p. 855-922. doi:10.1016/B978-0-444-59425-9.00028-7

van Mourik, C.A \& Brinkhuis, H. 2000. Data report: organic walled dinoflagellate cyst biostratigraphy of the latest middle to late Eocene at Hole 1053A (Subtropical Atlantic Ocean). Proceedings of the Ocean Drilling Program, 171B:1-25.

Warnaar, J.; Bijl, P.K.; Huber, M.; Sloan, L.; Brinkhuis, H.; Röhl, U.; Sriver, R. \& Visscher, H. 2009. Orbitally forced climate changes in the Tasman sector during the Middle Eocene. Palaeogeography, Palaeoclimatology, Palaeoecology, 280:361370. doi:10.1016/j.palaeo.2009.06.023

Wilson, G.J. 1967. Some new species of Lower Tertiary dinoflagellates from McMurdo Sound, Antarctica. New Zealand Journal of Botany, 5:57-83. doi:10.1080/002882 $5 X .1967 .10428735$

Wrenn, J.H. \& Beckman, S.W. 1982. Maceral, total organic carbon, and palynological analyses of Ross Ice Shelf Project site J9 cores. Science, 216:187-189. doi:10.1126/science.216.4542.187

Wrenn, J.H. \& Hart, G.F. 1988. Paleogene dinoflagellate cyst biostratigraphy of Seymour Island, Antarctica. Geological Society of America Memoirs, 169:321-447. doi:10.1130/ MEM169-p321

Zachos, J.C.; Dickens, G.R. \& Zeebe, R.E. 2008. An early Cenozoic perspective on greenhouse warming and carbon-cycle dynamics. Nature, 451:279-283. doi: 10.1038/nature06588

Zonneveld, K.A. et al. 2013. Atlas of modern dinoflagellate cyst distribution based on 2405 data points. Review of Palaeobotany and Palynology, 191:1-197. doi:10.1016/j.revpalbo.2012.08.003

Zonneveld, K.A.; Susek, E. \& Fischer, G. 2010. Seasonal variability of the organic-walled dinoflagellate cyst production in the coastal upwelling region off Cape Blanc (Mauritania): a five-year survey. Journal of Phycology, 46:202-215. doi:10.1111/j.15298817.2009.00799.x

Received in January, 2015; accepted in August, 2015. 